\title{
EFEITOS SISTÊMICOS DO VINAGRE DE MAÇÃ EM RATOS SUBMETIDOS À DIETA HIPERCALÓRICA VINAGRE DE MAÇÃ EM RATOS OBESOS
}

\section{SYSTEMIC EFFECTS OF APPLE VINEGAR IN RATS SUBMITTED TO THE HYPERCALORIC DIET APPLE VINEGAR IN OBESE RATS}

Gabriel Antonio Coltro', Cynthia Fontoura Klas', Rebeca Loureiro Rebouças*1, Cindy Caetano da Silva', Gabriel Abrahão Stoliar', Guilherme Cecchetti', Allan Giovanini', Camila Moraes Marques ${ }^{2,3}$, Luiz Fernando Kubrusly'.

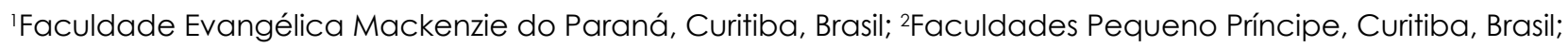
${ }^{3}$ Instituto de Pesquisa Pelé Pequeno Príncipe, Curitiba, Brasil.

*Autor correspondente: Endereço: Rua Padre Anchieta. 2770 - Bigorrilho, Curitiba -PR, 80730-000 Telefone: (41) 9 9763-9717 Email: rebecareboucas@gmail.com

\section{RESUMO}

A obesidade é um problema de saúde pública ao observar as projeções de incidência dessa doença nos próximos anos é possível verificar que os números são alarmantes. Diante do exposto, pode-se inferir que essa é uma enfermidade em que a condição pode estar associada ao desenvolvimento de síndrome metabólica, caracterizada pelo acúmulo excessivo de gordura em tecido adiposo. Em vista disso, se busca tratamentos para obesidade, nesse sentido, tem sido difundida a prática de ingestão diária de vinagre de maçã com intuito terapêutico, devido à potencial propriedade antihiperlipidêmica do ácido acético. Sendo assim, este trabalho tem como objetivo avaliar o efeito benéfico do uso de vinagre de maçã utilizando o modelo experimental de dieta hipercalórica em ratos. Para isso, foram utilizados 32 wistar machos divididos em 4 grupos: Grupo Controle (C), Grupo com administração de vinagre de maçã (V), Grupo com administração de dieta hipercalórica (D), Grupo com administração de dieta hipercalórica associada à administração de vinagre (DV). Durante o experimento, os grupos que tiveram obesidade induzida receberam dieta de cafeteria, enquanto que os demais grupos receberam ração. Após 3 meses, foi realizada a gavagem de vinagre de maçã por 8 semanas. Os animais foram sacrificados e foram retiradas amostras sanguíneas, gordura mesentérica, aorta e fígado. Após as análises não houveram diferenças significativas devido ao uso do vinagre. Portanto, o uso contínuo de vinagre de maçã em modelo animal não se mostrou efetivo para redução de peso, bem como na melhora dos parâmetros bioquímicos e histológicos.

Palavras-chave: Vinagre de maçã; dieta hipercalórica; ratos wistar; obesidade.

\begin{abstract}
Obesity is a public health problem. Observing the projections of the incidence of this disease over the coming years, it is possible to verify alarming numbers. In doing so, we can infer that obesity is a disease in which the condition may be associated with the development of metabolic syndrome, characterized by the excessive increase of fat in adipose tissue. In view of this, treatment for obesity is widely sought. The daily apple cider vinager consumption for therapeutic purposes has been increasingly widespread, due to a potencial antihiperlipidemic property of acetic acid. Therefore,
\end{abstract}


this work aims to evaluate the benefitial effect of consuming apple cider vinager by using the experimental high-calorie diet model in rats. For this study 32 males wistar were used divided into 4 groups: Control Group (C), Group with administration of apple cider vinegar (V), Group with administration of high calorie diet (D), Group with administration of high calorie diet associated with administration of vinegar (DV). During the experiment, the groups that had induced obesity received a diet in the cafeteria, while other groups received ration. After 3 months, apple cider vinegar was made for 8 weeks. The animals were sacrificed and removed from blood, mesenteric fat, aorta and liver. After the research, it was found that there were no significant differences among the studied groups due to the vinegar use. Therefore, the continuous use of apple cider vinegar in the animal model is not effective for reducing weight, as well as improving biochemical and histological parameters.

Keywords: Apple vinegar; hypercaloric diet; wistar rats; obesity.

\section{INTRODUÇÃO}

A obesidade se tornou uma das preocupações mais importantes de saúde pública do século XXI. Segundo o Ministério da Saúde o número de obesos no país aumentou 67,8\% entre 2006 e 2018. Muito disso se deve ao comportamento sedentário e à disponibilidade de escolhas alimentares altamente calóricas Brasil (2019), Halima et al (2017).

A consequência da dieta hipercalórica é a obesidade, esta é caracterizada pelo aumento da massa de tecido adiposo, órgão ativo endócrino e secretório, e resulta em doenças cardíacas coronarianas Halima et al (2017).

As doenças cardiovasculares são as principais causas de mortalidade em todo o mundo, pois as concentrações de colesterol e triglicerídeos séricos são reguladas por fatores genéticos e pela dieta. Além disso, os alimentos que contém grandes quantidades de gordura saturada afetam desfavoravelmente o perfil lipídico sanguíneo Budak et al (2011).

Bouazza et al (2015) descreveu que uma dieta rica em gordura afeta adversamente a saúde de espécies humanas e animais. E ainda a hiperlipidemia é um fator avançado de estresse oxidativo que leva a disfunções endoteliais, enquanto que o aumento dos peróxidos lipídicos plasmáticos é geralmente acompanhado pela redução do sistema de defesa antioxidante enzimático e não enzimático.

O desequilíbrio no metabolismo de ácidos graxos em associação com o tecido adiposo, tecido hepático e inflamação sistêmica é são fatores cruciais que leva ao desenvolvimento de hiperlipidemia e outras causas de risco cardiovascular. Esse processo é caracterizado pelo acúmulo de triglicerídeos dentro de hepatócitos na forma de macro e microvesículas lipídicas com um prognóstico benigno e uma subsequente esteatohepatite não-alcoólica (NASH), que se caracteriza por relativa inflamação, levando a fibrose, cirrose e falência hepática Bouazza et al (2015).

Recentemente, o vinagre tem sido utilizado como um alimento medicinal porque o ácido acético, que é o principal ingrediente do vinagre, é relatado por sua atividade antihiperlipidêmica. Halima et al (2017) e Kondo et al (2009).

Polifenóis tem tido um papel importante na saúde humana, pois previne uma variedade de doenças, tais como: câncer, hipertensão, doença cardíaca e diabetes. Vinagres normalmente apresentam 
altas taxas de polifenóis, dentre eles o ácido clorogênico, o qual impede o dano ao DNA in vitro e demonstra um efeito protetor contra doenças cardiovasculares a partir da inibição da oxidação do LDL Budak et al (2011).

O vinagre é produzido a partir da fonte de carboidratos fermentados, como maçãs, uvas e melaço. É comumente utilizado como conservante de alimentos, também como temperos em diferentes dietas, além disso é usado como um condimento e na medicina tradicional em todo o mundo Bouazza et al (2015). O principal componente do vinagre é o ácido acético, o qual está presente em concentrações de 3-5\%. Outros constituintes do vinagre incluem algumas vitaminas, sais minerais, aminoácidos, compostos polifenólicos e ácidos orgânicos Shishehbor et al (2008).

Resultados anteriores mostraram que o vinagre e o ácido acético (o principal ingrediente do vinagre) afetam o perfil lipídico e a perda de peso Bounihi et al (2016). Segundo Budak et al (2011), foram observados efeitos positivos de diversos vinagres na saúde, como aumento da absorção intestinal de cálcio, redução da pressão arterial e efeitos positivos na resposta da glicose sanguínea à insulina.

Mediante o exposto, este trabalho tem como objetivo avaliar o efeito benéfico do uso de vinagre de maçã, para isso foi utilizado o modelo experimental de dieta hipercalórica em ratos.

\section{METODOLOGIA}

\section{DELINEAMENTO EXPERIMENTAL}

Este estudo é de caráter experimental qualitativo-quantitativo. Os procedimentos com os animais estão em conformidade com o recomendado pela Comissão de Ética em Uso de Animais (CEUA) da Faculdade Evangélica Mackenzie do Paraná (FEMPAR), registrado sob o número 1605/2018.

\section{ANIMAIS}

Foram utilizados 32 ratos machos Wistar, pesando entre 200 e 300 gramas, provenientes do Biotério Central da Universidade Federal de Santa Catarina (UFSC - CIAEP/CONCEA No: 01.0127.2014). Os animais foram mantidos, durante o experimento, no biotério do Instituto de Pesquisas Médicas (IPEM) em caixas plásticas, de 47x34x18 cm, forradas com maravalha, em ciclo de doze horas claro/ escuro (luz das 7 às 19 horas) e temperatura de $22 \pm 2{ }^{\circ} \mathrm{C}$.

\section{VINAGRE DE MAÇÃ}

Foi ofertado vinagre de maçã vendido comercialmente, com ingredientes fermentado acético de maçã e água. Acidez 4,2\%. Foi utilizada a mesma marca durante o experimento.

\section{PARÂMETROS HISTOLÓGICOS}

Para a análise do fígado foram avaliadas a presença e a eventual graduação de esteatose, assim como a presença de balonização. Já para averiguar a aorta, o grau de lesão foi avaliado segundo os parâmetros da American Heart Association. Para análise da gordura mesentérica foram examinadas alterações dos adipócitos quanto ao número, diâmetro, volume, presença de fibrose e de macrófagos. 


\section{GRUPOS EXPERIMENTAIS}

O modelo utilizado foi o de administração de vinagre de maçã pelo método de gavagem, segundo Budak et al (2011). Os animais foram divididos em 4 grupos, com oito animais no grupo controle e oito animais em cada grupo experimental:

Grupo Controle (C) -Grupo não submetido à administração de vinagre de maçã, e com administração de veículo $(\mathrm{H} 2 \mathrm{O})$ por gavagem do $61^{\circ}$ dia ao $104^{\circ}$ dia;

Grupo com administração de vinagre de maçã (V) - Grupo não submetido à administração de dieta de cafeteria, apenas com administração de vinagre de maçã por gavagem do $61^{\circ}$ dia ao $104^{\circ}$ dia;

Grupo com administração de dieta hipercalórica (D) - Grupo submetido à dieta de cafeteria do $1^{\circ}$ ao $104^{\circ}$ dia e administração de veículo (H2O) por gavagem do $61^{\circ}$ dia ao $104^{\circ}$ dia;

Grupo com administração de dieta hipercalórica associada à administração de vinagre de maçã (DV) - Grupo submetido à administração de dieta de cafeteria do $1^{\circ}$ ao $104^{\circ}$ dia associado a administração de vinagre de maçã por gavagem do $61^{\circ}$ dia ao $104^{\circ}$ dia.

\section{PROCEDIMENTOS EXPERIMENTAIS}

\section{Modelo experimental de obesidade}

O modelo utilizado para indução de obesidade foi o da dieta de cafeteria (CAF) Gomez-smith et al (2016). Sendo assim, foi disponibilizado aos animais ração usual ad libitum e uma seleção diária de alimentos de mercearia selecionados de uma lista de dezesseis itens (TABELA 1), adaptada de Almeida et al (2008).

Tabela 1 - Alimentação dos animais com dieta de cafeteria

\begin{tabular}{ll} 
Domingo & Pão francês, bolacha recheada, marshmallow, mortadela e ração \\
\hline $\begin{array}{l}\text { Segunda-feira } \\
\text { Terça-feira }\end{array}$ & Bolacha waffer, paçoca, salsicha, brownie e ração \\
Quarta-feira & Cheetos cheese, marshmallow, hambúrguer, chocolate em barra e ração \\
Quinta-feira & Pão francês, mortadela, paçoca, bolacha waffer e ração \\
Sexta-feira & Bolacha recheada, salsicha, cheetos cheese, brownie e ração \\
Sábado & Pão francês, mortadela, marshmellow, bolacha, waffer e ração \\
\hline
\end{tabular}

Fonte: Adaptado de Almeida et al (2008)

Além disso, aos grupos de dieta hipercalórica foi disponibilizado, uma garrafa só com água e outra contendo água com sacarose a $12 \%$, afim de mimetizar os refrigerantes. Os animais foram submetidos a estes procedimentos por três meses. Este tempo foi necessário para o desenvolvimento de obesidade/síndrome metabólica Gomez-smith et al (2016). Os ratos também foram pesados semanalmente de acordo com técnica descrita de acordo com Budak et al, 2011

\section{Suplementação de vinagre de maçã e de veículo por gavagem}

Durante sete semanas, foi diministrado nos ratos dos grupos V e DV $1 \mathrm{ml}$ de vinagre de maçã diluído por gavagem oral $(157 \mu \mathrm{L}$ de vinagre de maçã em $843 \mu \mathrm{L}$ de água destilada, tal divisão foi usada para obter $1 \%$ de acidez total afim de evitar excesso de ácido acético). Nos demais grupos foi administrado volume proporcional de veículo (1 mL de água). 


\section{Eutanásia, obtenção de tecidos e amostras de sangue}

Após 104 dias de experimento, os animais foram pesados e anestesiados com uma mistura de Cloridrato de Xilasina 10mg/kg de peso corporal e Cloridrato de Cetamina $90 \mathrm{mg} / \mathrm{kg}$ de peso corporal.

Em seguida, foi realizada a eutanásia por meio da decaptação com auxílio de guilhotina para coleta de sangue, após tricotomia e desinfecção da região abdominal, foram extraídos os seguintes órgãos: fígado, gordura mesentérica e aorta torácica retirada a partir do coração.

Todos os órgãos foram pesados através do uso de uma balança de precisão, medidos (diâmetro e volume) e, imediatamente após a coleta, colocados em formol neutro tamponado, onde permaneceram por no mínimo 72 horas.

\section{Avaliação histológica}

Para realização do processo histológico, os órgãos coletados foram fixados em formol a 10\% dissolvido em 0,1M de PBS e pH 7,4. Em seguida, retirados fragmentos para processamento com base na técnica histológica convencional. Os fragmentos de fígado, gordura mesentérica, a aorta torácica foram incluídos em paraplast e orientados para que os cortes obtidos resultassem em secções transversais do órgão. Para analisar as alterações histopatológicas, os cortes foram chforados em hematoxilina-eosina.

\section{Análise laboratorial}

A partir da amostra de sangue coletada após a decapitação, foram avaliados: colesterol total, LDL, HDL, VLDL, triglicerídeos, glicemia. Além disso, foram feitos testes de integridade hepática: transaminase glutâmico-oxalacética (TGO); transaminase glutâmico-pirúvica (TGP).

\section{Análise estatística}

A partir dos dados coletados, as médias e os erros padrões das médias de cada grupo foram calculadas, utilizando para a investigação estatística o software Graphpad Instat, versão 3.0. Foi realizada análise de variância (ANOVA) seguida de teste Tukey-Kramer para múltiplas comparações, sendo o nível de significância adotado de $5 \%(\mathrm{P}<0,05)$. 


\section{RESULTADOS}

\section{EXAMES LABORATORIAIS}

\section{TGP, TGO e Colesterol Total}

Foi encontrada diferença estatisticamente significativa quando comparados os valores de TGP (Gráfico 1), TGO (Gráfico 2) e Colesterol Total entre os grupos C e V, D e V e V e DV. As demais comparações não apresentaram significância estatística $(\mathrm{P}>0,05)$.

Gráfico 1 - Efeitos de dieta hipercalórica e vinagre de maçã na TGP dos animais

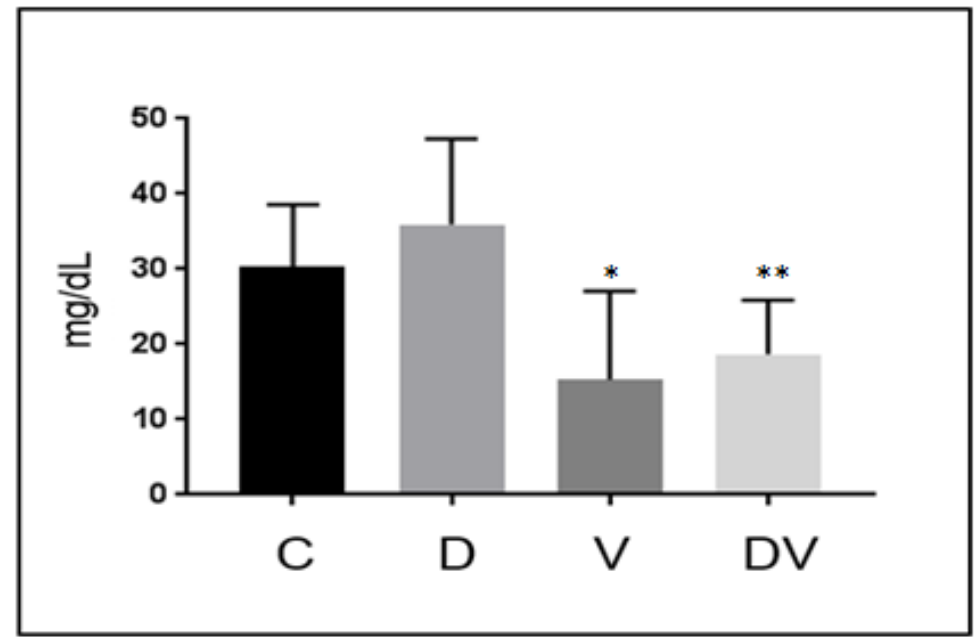

*Diferença significativa quando comparado aos grupos C e D

** Diferença significativa quando comparado ao grupo $\mathrm{V}$

Gráfico 2 - Efeitos de dieta hipercalórica e vinagre de maçã na TGO dos animais

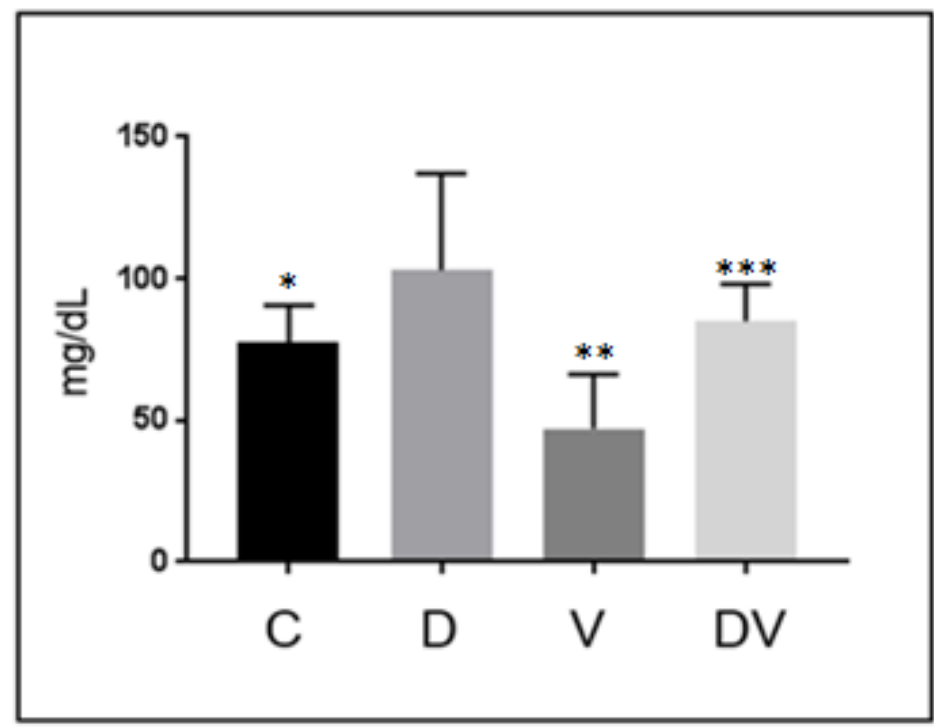

*Diferença significativa quando comparado ao grupo D ** Diferença significativa quando comparado ao grupo C *** Diferença significativa quando comparado ao grupo $\mathrm{V}$ 


\section{Glicose}

Os resultados apontaram diferença estatisticamente significativa quando comparamos os valores de glicose entre os grupos C e D ( $\mathrm{P}=0,012)$, C e DV ( $\mathrm{P}=0,0003)$, D e V ( $\mathrm{P}=0,018)$, V e DV $(\mathrm{P}=0,004)$. As demais comparações não apresentaram significância estatística (P > 0,05).

HDL

A diferença estatisticamente significativa foi encontrada quando comparados os valores de HDL entre os grupos C e D ( $\mathrm{P}=0,0099)$, D e V $(\mathrm{P}=0,0007)$ e V e DV ( $\mathrm{P}=0,0057)$. As demais comparações não apresentaram significância estatística $(\mathrm{P}>0,05)$.

\section{LDL, VLDL e Triglicerídeos}

Não foi encontrada diferença estatisticamente significativa entre os grupos $(P>0,05)$.

\section{PESOS}

\section{Pesos dos animais}

Para a variável peso (Gráfico 3), foi realizada a análise através do teste ordinary one-way, sem diferença significativa entre os grupos $(\mathrm{P}=0.5563)$. Foram também feitas múltiplas comparações entre dois grupos através do teste de Tukey, também não foram encontradas diferenças estatísticas entre os grupos $(\mathrm{P}>0,05)$.

E ainda foi o teste two-way, onde correlacionamos se o tempo e a dieta tiveram interferência sobre os grupos. A partir da sétima semana ocorreu diferença significativa entre o peso do grupo C e o grupo DV. A interação do teste no tempo foi de 36 e corresponde a $2.79 \%$ da variância total, com $\mathrm{F}=2.84, \mathrm{DFn}=36, \mathrm{DFd}=336$ e $\mathrm{P}$ valor $<0.0001$, com interação temporal considerada estatisticamente significante quando acompanhado com a dieta.

Gráfico 3 - Efeitos de dieta hipercalórica e vinagre de maçã no peso semanal dos animais

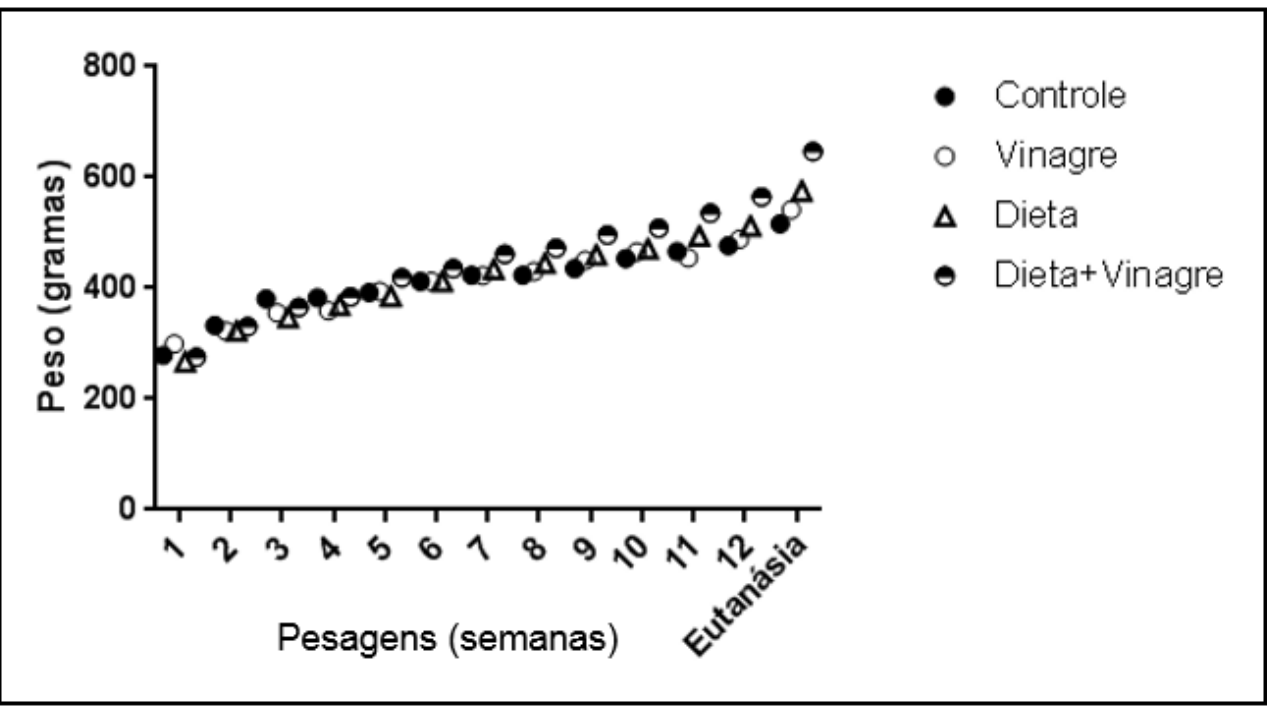




\section{Pesos dos órgãos}

Quanto a variável o peso do fígado teve diferença significativa, apenas entre o grupo V comparado ao grupo DV $(\mathrm{P}=0,0416)$. Já o peso da Aorta dos animais não foi observado nenhum valor estatisticamente significante $(\mathrm{P}>0,05)$. Com relação ao peso da gordura mesentérica é observado dado estatisticamente significante para: C e D ( $\mathrm{P}=0.0408)$; C e DV ( $\mathrm{P}=0,0002)$; V e D (P = 0,0046); V e DV $(\mathrm{P}<0,0001)$.

\section{LÂMINAS HISTOLÓGICAS}

\section{Fígado}

Os animais dos grupos D e DV tiveram esteatose hepática não alcoólica (NASH), balonização de hepatócitos sem a presença de fibrose, sendo que o grau de esteatose foi maior no grupo D em relação ao grupo DV. Associado a isso, houve perda dos capilares sinusoides em 5 dentre 8 lâminas no grupo D. De um total de 8 lâminas, seis apresentaram estatose grau 3 (maior que 66\% dos hepatócitos acometidos), e duas lâminas apresentaram grau 1 (entre 5 e 33\% dos hepatócitos acometidos), de acordo com a classificação de Kleinert et al. (2005). Os grupos C e V não tiveram alterações na histologia hepática, sem esteatose ou outras alterações nos hepatócitos ou sistema vascular hepático.

\section{Aorta}

Não foi possível observar alterações compatíveis com aterosclerose nas camadas da aorta torácica. Não ocorreu também a formação de trombose ou qualquer alteração endotelial em todos os grupos.

\section{Gordura mesentérica}

Não foi possível observar alterações histológicas na gordura mesentérica de todos os grupos. Alterações de vascularização ou calcificação também não foram observadas.

\section{DISCUSSÃO}

Neste estudo, em comparação realizada entre os grupos C e D, foi demonstrada diferença significativa entre os níveis de HDL, com um aumento de seus valores no grupo D, dado também encontrado no estudo de Budak et al (2011), no qual foi constatado um aumento significativo nos níveis de HDL, LDL, Triglicerídeos e VLDL no grupo em que foi instituída dieta com altas taxas de colesterol em comparação ao grupo controle, demonstrando efeito de hiperlipidemia da dieta hipercalórica.

No entanto, divergindo desses dados, especificamente em relação ao HDL, Bounihi et al (2016), em estudo semelhante, encontrou redução de 20,1\% na concentração plasmática de HDL em ratos submetidos a dieta hipercalórica quando comparados a ratos alimentados com dieta balanceada (não submetidos à dieta hipercalórica).

Shishehbor et al (2008) encontrou uma redução de 47\% dos níveis de LDL e um aumento de 34\% dos níveis de HDL em ratos normais tratados com vinagre de maçã, com diferença estatisticamente significante entre este grupo e o grupo controle. Diferente da literatura, no presente estudo, não houve diferença significante entre os grupos C e V nas taxas de HDL e LDL. 
Em estudo semelhante de Bouderbala et al (2016), na comparação de grupos dieta hipercalórica e dieta hipercalórica associada a administração de vinagre de maçã, foi encontrada redução plasmática significativa dos níveis de TG, LDL e VLDL no segundo grupo, associada ao aumento dos níveis de HDL, dados também encontrados por Bounihi et al (2016), já em relação aos níveis de Triglicerídeos e LDL demonstraram oferecer proteção contra risco aterogênico. Todavia, no atual estudo, não houve diferença significativa entre essas variáveis de um para o outro nos grupos D e DV.

Bounihi et al (2016) também constataram, após tratamento com vinagre de frutas, que a concentração plasmática de HDL em ratos submetidos à dieta hipercalórica elevou-se para valores próximos aos encontrados em ratos alimentados apenas com dieta balanceada. Diferentemente do encontrado no presente estudo, no qual não houve diferenças significativas entre os níveis de HDL dos grupos DV e C.

A dieta hipercalórica resultou no aumento significativo do colesterol total sérico se comparados aos grupos que receberam dieta e os demais, assim como no estudo feito por Budak et al. (2011). Porém, não houve distinção significativa entre os grupos D e DV, corroborando com que o vinagre de maçã não tenha efeito com real significância para baixar os níveis de colesterol total, a partir de níveis elevados já estabelecidos por uma dieta hipercalórica ou qualquer outra razão para o aumento de colesterol. Budak e demais autores também não encontraram diferença significativa entre os grupos que receberam vinagre e dieta hipercalórica e o grupo que recebeu apenas a dieta rica em gordura.

No estudo em questão, a administração do vinagre de maçã diminui a função hepática assim como descrito por Bounihi et al. (2015). A análise dos valores de TGP e TGO demonstra uma diferença significativa entre o grupo que recebeu vinagre isoladamente dos demais grupos. A hiperlipidemia causa estresse oxidativo, reduzindo o sistema de defesa antioxidante e elevando os peróxidos lipídicos Vijayaraj et al (2013). Como o estresse oxidativo é um dos principais fatores que contribui para a ocorrência de problemas hepáticos, vários estudos indicaram que os antioxidantes podem impedir a extensão da peroxidação lipídica Laurent et al (2004). No estudo em questão, não houve diferença significativa entre os grupos DV e D, apesar do grupo que recebeu vinagre e dieta ter valores menores das enzimas referentes à inflamação hepática se comparado ao grupo que recebeu só dieta. Pode-se considerar que o vinagre é eficiente em seres que recebem dieta normocalórica, funcionando como uma prevenção da elevação das enzimas hepáticas por qualquer que seja a patologia desencadeante.

Os níveis séricos de glicose foram significativamente mais altos nos grupos que receberam dieta hipercalórica em relação ao grupo controle, confirmando o resultado obtido por Budak et al. (2011). Porém, ao comparar os grupos DV e D, não houve diferença significativa, mais uma vez, como no caso do colesterol, o vinagre de maçã não foi efetivo para os níveis altos já estabelecidos. Os resultados obtidos no presente estudo são semelhantes ao estudo feito por Budak et al. (2011), porém são distintos dos resultados em estudo feito por Ebihara e Nakajima (1988), em que os grupos que receberam o vinagre de maçã tiveram uma redução de 30\% dos níveis de glicose. Uma hipótese sugerida por estes autores perante esse resultado seria que o ácido acético levaria a um atraso no esvaziamento gástrico. Contudo recomenda-se que novos estudos devem ser feitos para melhor interpretação do efeito do vinagre de maçã nos níveis séricos de glicose no sangue.

O estudo presente não encontra diferenças significativas atribuídas ao uso do vinagre quanto ao peso dos animais utilizados. Tal dado vai de encontro ao trabalho publicado por Bouderbala e colaboradores Bouderbala et al (2016)ou ayant des effets secondaires minimes, est un défi. Cette étude vise à évaluer l'effet anti-obésogène du vinaigre de cidre de pomme (VCP, o qual utilizou da 
mesma metodologia, por meio de engorda por dieta de cafeteria, e encontrou diferença significativa na perda de peso entre os grupos. Diferenças significativas foram encontradas quando comparados os grupos controle e dieta com o grupo dieta + vinagre, o qual teve maior média de peso dentre os grupos. Da mesma forma, Soltan e Shehata demonstraram em um artigo alteração significativa do peso com aplicação do vinagre de frutas durante 7 meses Soltan e Shehata (2012)

Kondo e colaboradores Kondo et al (2009), em estudo realizado com humanos, também citam a diferença estatisticamente significativa do vinagre para reduzir o peso. Isso se deve à propriedade do ácido acético de inibir a expressão de genes lipogênicos, entre eles, o ácido-graxo sintetase e acetil CoA carboxylase por meio da proteína quinase AMP-ativada (AMPK) / proteína ligada ao elemento de resposta aos carboidratos, o que resultaria na diminuição do peso em ratos obesos.

Bouazza e colaboradores Bouazza et al (2016), observando os efeitos de vários vinagres de frutas na redução do peso, demonstram achados similares aos resultados desse estudo, com ausência de diferença significativa nos pesos dos ratos atribuídos ao vinagre.

Bouderbala também cita que a adiposidade central diminuiu de maneira estatisticamente significante, do mesmo modo atribuiu a inibição da lipogênese Bouderbala et al (2016)ou ayant des effets secondaires minimes, est un défi. Cette étude vise à évaluer l'effet anti-obésogène du vinaigre de cidre de pomme (VCP. Em nosso estudo, quando investigado o peso da gordura abdominal, observa-se diferença estatística entre todos os grupos, não podendo atribuir tal efeito ao vinagre.

Também são relatadas reduções estatisticamente significantes no processo aterosclerótico e no dano hepático em grupos de ratos tratados com vinagre Bouderbala et al (2016); Kondo et al (2009). Quanto aos pesos do fígado, observou-se diferença significativa ao comparar o grupo V com o grupo DV apenas. Quanto ao peso da aorta torácica dos animaisnão foi encontrada nenhuma diferença entre os grupos.

O grupo que recebeu apenas a dieta hipercalórica teve um aumento significativo da esteatose hepática se comparado aos demais grupos, assim como no estudo feito por Budak et al. (2011). Segundo esse mesmo estudo, o vinagre de maçã reduziu os níveis de esteatose hepática significativamente, o que não corrobora com o encontrado em nosso estudo.

\section{CONCLUSÃO}

O uso contínuo de vinagre de maçã em modelo animal não se mostrou efetivo para redução de peso, bem como na melhora dos parâmetros bioquímicos e histológicos. Portanto, sugere-se que o uso de vinagre de maçã não exerça efeitos benéficos envolvidos no emagrecimento.

\section{REFERÊNCIAS}

ALMEIDA, F. N. et al. A resposta do peso e da composição corporal à inclusão da dieta de cafeteria e treinamento físico aeróbio em diferentes fases do desenvolvimento. Cienc Cuid Saude, v. 7, n. 1, p. 39-44, 2008.

BOUAZZA, A. et al. Effect of fruit vinegars on liver damage and oxidative stress in high-fat-fed rats.

Pharm Biol, Early Online: 1-6, 2015. 
BOUDERBALA, H. et al. Effet anti-obésogène du vinaigre de cidre de pomme chez le rat soumis à un régime hyperlipidique. Annales de Cardiologie et d'Angéiologie, v. 65, n. 3, p. 208-213, 1 jun. 2016.

BOUNIHI, A. et al. Fruit vinegars attenuate cardiac injury via anti-inflammatory and antiadiposity actions in high-fat diet-induced obese rats. Pharmaceutical Biology, 2016.

BRASIL. Ministério da Saúde. Agência Nacional de Saúde. Dados do setor. Brasília, 2019. Disponível em: https://www.saude.gov.br/noticias/agencia-saude/45612-brasileiros-atingem-maior-indice-deobesidade-nos-ultimos-treze-anos. Acesso em: 08 jul. 2019.

BUDAK, N.H. et al. Effects of Apple Cider Vinegars Produced with Different Techniques on Blood Lipids in High-Cholesterol-Fed Rats. J. Agric. Food Chem. 201 1, 59, 6638-6644.

EBIHARA, Kiyoshi; NAKAJIMA, Akira. Effect of acetic acid and vinegar on blood glucose and insulin responses to orally administered sucrose and starch. Agricultural and biological chemistry, v. 52, n. 5, p. 1311-1312, 1988.

GOMEZ-SMITH, M. et al. A physiological characterization of the Cafeteria diet model of metabolic syndrome in the rat. Physiology \& behavior, v. 167, p. 382-391, 2016.

HALIMA, Ben Hmad et al. Apple cider vinegar attenuates oxidative stress and reduces the risk of obesity in high-fat-fed male Wistar rats. Journal of medicinal food, v. 21, n. 1, p. 70-80, 2018.

KLEINER, David E. et al. Design and validation of a histological scoring system for nonalcoholic fatty liver disease. Hepatology, v. 41, n. 6, p. 1313-1321, 2005.

KONDO, T. et al. Vinegar Intake Reduces Body Weight, Body Fat Mass, and Serum Triglyceride Levels in Obese Japanese Subjects. Biosci Biotechnol Biochem, 73 (8), 1837-1843, 2009.

LAURENT, Alexis et al. Pivotal role of superoxide anion and beneficial effect of antioxidant molecules in murine steatohepatitis. Hepatology, v. 39, n. 5, p. 1277-1285, 2004.

SHISHEHBOR, F. et al. Apple Cider Vinegar Attenuates Lipid Profile in Normal and Diabetic Rats. Parkistan Journal of Biological Sciences, 11 (23): 2634-2638, 2008.

SOLTAN, S. S. A.; SHEHATA, M. M. E. M. Antidiabetic and hypocholesrolemic effect of different types of vinegar in rats. Life Science Journal, 2012.

VIJAYARAJ, Panneerselvam et al. Antihyperlipidemic activity of Cassia auriculata flowers in triton WR 1339 induced hyperlipidemic rats. Experimental and Toxicologic Pathology, v. 65, n. 1-2, p. 135141, 2013. 